\title{
Can Multiple IRBs Spoil the Broth?
}

I the federal rules governing research (the "Common Rule”), 45CFR46, Section 46.114 reads:

$\$ 46.114$ Cooperative research.

Cooperative research projects are those projects covered by this policy which involve more than one institution. In the conduct of cooperative research projects, each institution is responsible for safeguarding the rights and welfare of human subjects and for complying with this policy. With the approval of the department or agency head, an institution participating in a cooperative project may enter into a joint review arrangement, rely upon the review of another qualified IRB, or make similar arrangements for avoiding duplication of effort.

The federal rules cover any research funded by various federal agencies or any research on new drugs or devices. The issue with this language is that although it seeks to promote a single review, it requires every institution to be responsible for the welfare of human subjects. This has been understood by individual institutional review boards (IRBs) to mean that each must conduct it own full review. As more studies are done at numerous sites, this results in multiple IRB review of single protocols.

Ravina et al argue in their article on local IRB review of multicenter trials that individual reviews at multiple sites add substantial costs without substantial benefit. ${ }^{1}$ They estimate review costs of approximately $\$ 107,544$ in direct costs plus $\$ 82,610$ in fees and $\$ 24,934$ in labor. They show that individual reviews only resulted in minor, nonsignificant changes, such as the addition of each local IRB's preferred standard consent form language on standard clauses. There is no evidence that these minor changes make any difference in a subject's decision to participate. ${ }^{2}$

The authors found no important changes to the study protocol. They conclude that individual review results in significant expense and time for a very small return. $^{1}$

Because an important goal of IRB review is protection of human subjects, a central review seems better for a detailed safety and risk-benefit review. If a second goal is an understandable study with clear information to enable a prospective participant to decide whether to enroll, again a central review could best design an understandable standardized consent process and form. If a third goal is to be sure that local investigators are qualified and local enrollment approaches are not coercive, there would be a role for local review limited to local issues. However, the Ravina study shows that local IRBs do not have a major focus on local issues.

Local IRBs are increasingly overburdened, leaving them insufficient time to concentrate on significant local issues like participant recruitment, local researcher qualifications, and local safety. Use of a central IRB could allow local IRBs more time to concentrate on issues of local importance. $^{3}$

The US Food and Drug Administration (FDA) has issued guidance on using a centralized IRB for multicenter trials. ${ }^{4}$ The guideline points out the substantial growth over time in multicenter studies and the consequent needless duplication of effort by individual IRBs at each center, resulting in increased expenses. Although the FDA recognizes the need to consider local aspects of review, it suggests that a centralized IRB review could include consideration of local factors by consulting with the local IRB or allowing limited local IRB review.

IRBs ought to have sufficient time to review protocols to be sure human subjects are protected. They should not duplicate efforts of other equally qualified IRBs, nor should they impose their own versions of boilerplate language in consent forms on other IRBs. It would be preferable to establish a template for items like the injury clause, who to contact with questions, and how to withdraw from a study. Indeed, in cancer drug protocols, a central IRB should be in a better position to determine the safest way to withdraw, rather than having each local IRB write its own language.

It may be that the Office of Human Research Protection (OHRP) oversight has caused local IRBs to be nervous about ceding review to a central IRB, but if the goal is to protect subjects, then the proper local IRB analysis must focus on what protection is being lost by a central board review.

A full solution to overburdened IRBs will involve new OHRP rules, templates for consent forms, minimal 


\section{ANNALS of Neurology}

review for minimal-risk protocols, extended review periods, and other changes to the current system. Central review supported by the OHRP could, as the authors suggest, be an important improvement element. It would be useful for the OHRP to finalize its proposed rule change, making it easier for multicenter studies to rely on a central IRB review.

\section{Edward B. Goldman, JD}

Department of Obstetrics and Gynecology

University of Michigan Health System

Ann Arbor, MI

\section{References}

1. Ravina et al. Local IRB review of a multicenter trial: local costs without local context. Ann Neurol 2010;67:258-260.

2. Appelbaum PS, Lidz CW, Klitzman R. Voluntariness of consent to research: a preliminary empirical investigation. IRB 2009;31:10-14.

3. Kim $S$, Ubel $P$, De Vries R. Pruning the regulatory tree. Nature 2009;457:534-535.

4. Health and Human Services, Food and Drug Administration, Center for Drug Evaluation and Research. Regulatory Information, Using a Centralized IRTB Review Process in Multicenter Clinical Trials. March 2006. Available at:http://www.fda.gov/Regulatorylnformation/ Guidances/ucm 127004.htm Accessed Feb. 10, 2010.

DOI: 10.1002/ana.21999 